\title{
Características psicopatológicas y dimensiones de personalidad en los pacientes dependientes del alcohol: un estudio comparativo
}

\author{
Ricardo Bravo de Medina'; Enrique Echeburúa²; Javier Aizpiri ${ }^{1}$ \\ ${ }^{1}$ Medicina Psico-Orgánica, S.L. Bilbao \\ 2 Facultad de Psicología. Universidad del País Vasco. \\ Enviar correspondencia a: \\ Enrique Echeburúa. Facultad de Psicología. Avda. de Tolosa, 70. 20018 San Sebastián. E-mail: enrique.echeburua@ehu.es
}

Recibido: Julio de 2007

\section{RESUMEN}

El objetivo de este estudio fue describir las características psicopatológicas y dimensiones de personalidad de los pacientes dependientes del alcohol, comparándolas, a su vez, con un grupo de control clínico de pacientes psiquiátricos, pero sin diagnóstico de abuso de sustancias, y con un grupo de control sano. La muestra estuvo compuesta por 158 pacientes dependientes del alcohol en tratamiento ambulatorio, 120 pacientes no-adictos, también en tratamiento ambulatorio, y 103 sujetos sanos apareados con las muestras clínicas en sexo, edad y nivel socioeconómico. Todos los sujetos fueron evaluados con diferentes instrumentos relacionados con la personalidad (Escala de Búsqueda de Sensaciones, Escala de Impulsividad y Cuestionario de Ansiedad-Rasgo), con las características psicopatológicas (SCL-90-R) y con los trastornos de personalidad (MCMI-II). Los pacientes pertenecientes a los grupos clínicos mostraron una mayor sintomatología ansiosodepresiva y mayores problemas de inadaptación que los sujetos del grupo de control sano, pero no había diferencias entre los grupos clínicos. Los pacientes dependientes del alcohol presentaron un mayor nivel de impulsividad y de búsqueda de sensaciones que los otros dos grupos. Los trastornos de personalidad de los pacientes dependientes del alcohol fueron el histriónico, el narcisista y el antisocial. Por último, se comentan las implicaciones de esta investigación para la práctica clínica y para futuras investigaciones.

Palabras clave: dependencia del alcohol, comorbilidad, dimensiones de personalidad, características psicopatológicas.

\section{ABSTRACT}

The aim of this paper was to describe the psychopathological and personality profile associated with alcohol dependence and to compare it with those of non-addictive disorders and the normal population. The sample consisted of 158 alcoholdependent participants attending a psychiatric outpatient clinic, 120 psychiatric patients with non-addictive disorders and 103 participants from the general population chosen to match the patient samples for age, gender and socioeconomic level. All participants were assessed with different instruments related to personality (Impulsiveness Scale, Sensation-Seeking Scale and STAI), psychopathology (SCL-90-R, BDI and Maladjustment Scale) and personality disorders (MCMI-II). Patients from the clinical groups presented more symptoms of anxiety and depression than the healthy participants and had more problems adjusting to everyday life, but there were no differences between the two clinical groups. Alcohol-dependent patients were more impulsive and sensation-seeking than the other two groups. Histrionic, narcissistic and antisocial personality disorders were specific to the alcohol-dependent patients. The implications of this study for further research are discussed.

Key words: alcohol dependence, comorbidity, personality traits, psychopathological symptoms.

\section{INTRODUCCIÓN}

E la sociedad actual la dependencia y el abuso de alcohol constituyen un fenómeno psicopatológico de gran relevancia clínica y social. Las tasas de prevalencia tan elevadas que se observan en la población española se relacionan, probablemente, con la facilidad de acceso al alcohol, con su integración cultural, con su bajo precio y con sus propiedades psicoactivas (Bravo de Medina, Echeburúa y Aizpiri, en prensa; Fernández-Montalvo, Landa y López-Goñi, 2005). 
La comorbilidad psicopatológica en pacientes dependientes del alcohol representa en la actualidad un tema de interés clínico creciente. Cuando una persona es adicta al alcohol o a otra droga, en el 50-75\% de los casos presenta otro trastorno adicional (Casas, 1994; Ross, 1995). Esta cifra de comorbilidad psicopatológica está muy por encima de la tasa de prevalencia de trastornos mentales en la población general (15-20\%) e incluso de la de otro tipo de pacientes noadictos. De forma específica, la comorbilidad más alta de la dependencia del alcohol se da con los trastornos de ansiedad (19,4\%), con los trastornos afectivos $(13,4 \%)$ y con la esquizofrenia $(3,8 \%)$ (Casas y Guardia, 2002).

Por lo que se refiere específicamente a los trastornos de personalidad, la tasa de comorbilidad de la dependencia del alcohol oscila entre el $40 \%$ y el $44 \%$ de los casos estudiados. En concreto, la mayor comorbilidad aparece con los trastornos de la personalidad obsesivo-compulsivo, antisocial, paranoide y dependiente (Echeburúa, Bravo de Medina y Aizpiri, 2005, 2007; Fernández-Montalvo y Landa, 2003).

Pero más allá de las cifras tan elevadas, el estudio de la comorbilidad psicopatológica de la dependencia del alcohol tiene una significación clínica relevante. La comorbilidad está asociada a una mayor probabilidad de problemas psicosociales e interpersonales y a un mayor riesgo de suicidio, así como a una menor adherencia al tratamiento y a una mayor dificultad en el mantenimiento de la abstinencia (Haver, 2003; Holdcraft, Jacono y McGue, 1998; Kranzler, Mason y Modesto-Lowe, 1998; Rousanville, Dolinsky, Babor y Meyer, 1987).

Por ello, un mejor conocimiento de las dimensiones de personalidad y la detección y el tratamiento de los trastornos psicopatológicos asociados a la dependencia del alcohol desempeñan un papel importante en la investigación clínica porque influyen directamente en la evolución y en el pronóstico de la dependencia (Haver, 2003; Landa, Fernández-Montalvo, López-Goñi y Lorea, 2006).

El objetivo de esta investigación es llevar a cabo un análisis comparativo de las características psicopatológicas y de las dimensiones de personalidad en los pacientes dependientes del alcohol, comparándolos con un grupo de pacientes no adictos y con un grupo de control sano para conocer así el perfil de la comorbilidad en los pacientes dependientes del alcohol. Se trata de profundizar más en esta área para, posteriormente, establecer programas terapéuticos adaptados a las necesidades específicas de este tipo de pacientes y conseguir así una mayor eficacia terapéutica.

\section{MÉTODO}

\section{Participantes}

La muestra de esta investigación está compuesta por 381 sujetos, distribuidos en 3 grupos:

1) Grupo experimental $(N=158)$. Los criterios de inclusión fueron los siguientes: a) tener más de 18 años y buscar ayuda terapéutica por problemas relacionados con los trastornos por uso de alcohol; b) cumplir los criterios de dependencia alcohólica según el DSM-IV-TR (APA, 2000); y c) recibir el diagnóstico de dependencia del alcohol como diagnóstico primario.

2) Grupo de control clínico $(N=120)$. Se trata de pacientes con un trastorno mental y con un diagnóstico distinto del de dependencia del alcohol o de sustancias. La muestra de este grupo está constituida por pacientes que acuden al mismo servicio clínico.

3) Grupo de control sano $(N=103)$. Se trata de personas sanas, sin patología mental, apareadas en edad, sexo y nivel cultural con los grupos anteriores. La muestra de esta población se ha seleccionado entre la población que no recibe tratamiento psiquiátrico ni psicológico.

Todos los pacientes incluidos en el estudio fueron atendidos en la Unidad de Salud Mental de la Clínica de Medicina Psico-orgánica (Bilbao) entre 2003 y 2006 y dieron el consentimiento informado para formar parte de este estudio.

\section{Medidas de evaluación}

\section{a) Entrevista general}

Al principio se realizó una entrevista individual semiestructurada para realizar el diagnóstico clínico según los criterios del DSM-IV-TR. En esta entrevista se recogían los datos más significativos: motivo de consulta, trastorno actual, antecedentes, familia de origen, familia actual, historia personal, nivel académico y actividad laboral, vida familiar, relaciones sociales, hábitos de vida, consumo de otras drogas, etcétera.

\section{b) Pruebas específicas}

b1) Relacionadas con las dimensiones de la personalidad

Escala de Búsqueda de Sensaciones (SSS) (Zuckerman, Eysenck y Eysenck, 1978) (validada por Pérez y Torrubia, 1986). La escala está compuesta por 40 ítems, que se contestan de forma autoaplicada con la indicación de verdadero o falso. Cada respuesta afirmativa equivale a 1 punto y cada respuesta negativa 
a 0 . No existen puntos de corte propuestos. Cuanto mayor es la puntuación, mayor es la presencia del rasgo.

La escala recoge cuatro dimensiones, que son la búsqueda de emociones (BEM), la búsqueda de excitación (BEX), la desinhibición (DES) y la susceptibilidad al aburrimiento (SAB).

Escala de Impulsividad (Barratt, 1985; versión española de Luengo, Carrillo de la Peña y Otero, 1991). Se trata de una escala autoaplicada que consta de 33 ítems, con 5 opciones de respuesta, que puntúan de 0 a 4 (casi nunca/nunca, algunas veces, a veces sí) a veces no, bastantes veces, casi siempre/siempre). Según Barratt, se pueden precisar 3 tipos de impulsividad: la impulsividad motora, la impulsividad cognitiva y la improvisación/ausencia de planificación.

La puntuación total se obtiene sumando las puntuaciones obtenidas. No existe un punto de corte propuesto. Desde la perspectiva clínica, lo que va a tener mayor importancia es el valor cuantitativo de la puntuación total.

Cuestionario de Ansiedad-Rasgo (STAl-R). Es una parte del cuestionario de ansiedad estado-rasgo (Spielberg, Gorsuch y Lushene, 1970; versión española de TEA, 1982). Se valora la ansiedad-rasgo como una predisposición ansiosa estable a percibir las situaciones como amenazantes. Consta de 20 ítems, que miden la ansiedad como rasgo, según una escala de tipo Likert que va de 0 a 3 (nada, algo, bastante, mucho). La puntuación total oscilará entre 0 y 60 . El punto de corte para la población masculina es de 25 y para la población femenina de 32.

La versión española de esta prueba presenta unos índices de consistencia interna para la $A-R$ situados entre 0,84 y 0,87 . Los valores de fiabilidad calculada por el procedimiento de las dos mitades alcanzan un valor de 0,86.

b2) Relacionadas con las características psicopatológicas

Symptom Checklist-90-Revised (SCL-90-R) (Derogatis, 1975; adaptación española de González de Rivera, 2002). Es un cuestionario autoaplicado multidimensional compuesto por 90 ítems. El cuestionario proporciona información en relación a nueve dimensiones de sintomatología psicopatológica (somatización, obsesivo-compulsiva, sensibilidad interpersonal, depresión, ansiedad, hostilidad, ansiedad fóbica, ideación paranoide y psicoticismo), así como a tres escalas generales, que son el Índice Global de Severidad (GSI), el Índice de Distrés de Síntomas Positivos (PST) y el Índice de Sintomatología Positiva Total (PSDI).
Existen datos normativos españoles tanto para la población clínica como para la general. Los índices alfa de fiabilidad de la adaptación española del SCL90- $R$ están entre 0,77 y 0,90. El SCL-90-R muestra una elevada sensibilidad diagnóstica, de entre el 80 y el $90 \%$, pero una muy baja especificidad, ente el 20 y el $60 \%$.

Cuestionario de Ansiedad-Estado (STA/-E). Deriva del cuestionario de ansiedad estado-rasgo (Spielberger, Gorsuch y Lushene, 1970; versión española de TEA, 1982). Se valora un estado emocional transitorio, caracterizado por sentimientos subjetivos, conscientemente percibidos, de atención tensa y aprensión y por hiperactividad del Sistema Nervioso Autónomo.

Se trata, como en el caso de la valoración de la ansiedad-rasgo, de 20 frases con 4 alternativas de respuestas, que van de 0 a 3 (nada, algo, bastante, mucho), en donde el sujeto muestra cómo se siente "ahora mismo". El rango es de 0 a 60. El punto de corte para la población masculina es de 28 y para la población femenina de 31 .

La versión española de esta prueba presenta unos índices de consistencia interna para la A-E situados entre 0,90 y 0,93. Los valores de fiabilidad calculada por el procedimiento de las dos mitades alcanzan un valor de 0,94.

Inventario de Depresión de Beck (BD/). Es un autoinforme que evalúa la presencia e intensidad de sintomatología depresiva (Beck, Rush, Shaw y Emery, 1979) y está validado para la población española (Sanz y Vázquez, 1998).

La versión utilizada consta de 21 apartados con diferentes opciones de respuesta (entre 4 y 8), cada una de las cuales tiene un valor entre 0 y 3 , en donde 0 representa la ausencia de sintomatología y 3 la máxima gravedad. Se toman en consideración las siguientes puntuaciones: 0-9, ausencia de depresión; 10-18, sintomatología depresiva leve; 19-29 sintomatología depresiva moderada; e igual o mayor que 30, sintomatología depresiva grave.

El $B D /$ posee unas buenas propiedades psicométricas, con una fiabilidad media de coeficiente alfa de 0,86 y 0,74, según Beck, Steer y Garbin (1988). El BDI presenta una fiabilidad alta en términos de consistencia interna (alfa total de la escala $=0,90$ ). Con un punto de corte de 21 , la sensibilidad es del $75 \%$ y la especificidad del 76\% (Richter, Werner, Heerlein, Graus y Saber, 1989; Weis, Griffin y Mirin, 1989; Vázquez y Sanz, 1999).

Escala de Inadaptación (El) (Echeburúa, Corral y Fernández-Montalvo, 2000). Es un instrumento de autoevaluación que consta de seis ítems, que se valoran de 0 a 5 puntos en una escala de tipo Likert. En 
esta escala se recogen cuatro aspectos referentes a la inadaptación en la vida cotidiana: trabajo/estudios, vida social, tiempo libre, relaciones de pareja y vida familiar.

Las puntuaciones totales pueden oscilar entre $0 \mathrm{y}$ 30 , estableciéndose como puntos de corte la puntuación 12 en la escala total y la de 2 en cada uno de los ítems para discriminar los niveles clínicamente significativos de inadaptación. Las propiedades psicométricas son satisfactorias (Echeburúa et al., 2000).

b3) Relacionadas con los trastornos de personalidad

El Millon Clinical Multiaxial Inventory-// (MCMI-/l) (Millon, 1997) es un cuestionario clínico autoaplicado que consta de 175 ítems de respuesta dicotómica y tiene como objetivo poder identificar trastornos de personalidad. Proporciona información sobre 10 escalas básicas de personalidad (1 Esquizoide, 2 Evitativa, 3 Dependiente, 4 Histriónica, 5 Narcisista, 6A Antisocial, 6B Agresivo-Sádica, 7 Compulsiva, 8A PasivoAgresiva y 8B Autodestructiva) y sobre 3 escalas de personalidad patológica (S Esquizotípica, C Límite, P Paranoide).

El punto de corte, siguiendo los criterios más conservadores de Weltzler (1990), se ha establecido en las puntuaciones cuya tasa base sea mayor que 84 (TB>84). La corrección final se realiza de forma informatizada mediante los disquetes de corrección de TEA.

La validación española del MCMI-// para las diferentes escalas obtiene unos índices alfa de fiabilidad (consistencia interna) altos, que oscilan entre 0,70 y 0,90 , excepto para las escalas Esquizoide $(0,68)$, Dependiente $(0,68)$ y Compulsiva $(0,63)$.

\section{Procedimiento}

El diagnóstico de dependencia del alcohol ha sido llevado a cabo, con arreglo a los criterios específicos del DSM-IV-TR, por un psiquiatra y por un psicólogo clínico conjuntamente, ambos con una amplia experiencia clínica. El grado de concordancia entre ambos profesionales respecto al diagnóstico fue del 100\%.

Por lo que se refiere a las dimensiones de personalidad y a los síntomas psicopatológicos, una vez seleccionados todos los sujetos de la muestra, se les administró la batería de pruebas en 2-3 sesiones. La administración de las pruebas se llevó a cabo, en todos los casos, inmediatamente después de la fase de desintoxicación, cuando todos los sujetos estaban abstinentes.

Para el análisis de los resultados se han utilizado tablas de frecuencia y pruebas paramétricas y no paramétricas para muestras independientes. La comparación de variables cuantitativas se ha realizado mediante análisis de la varianza. Como análisis posthoc se ha utilizado la prueba de Bonferroni. Estos resultados se han obtenido por medio del SPSS 13.0 para Windows.

\section{RESULTADOS}

En este trabajo se han analizado los siguientes datos en el conjunto de la muestra: a) las variables sociodemográficas; b) las características psicopatológicas; c) las dimensiones de personalidad; y d) los trastornos de personalidad.

\section{Variables sociodemográficas}

Las principales características sociodemográficas de la muestra están señaladas en la tabla 1.

Como se puede observar, la única diferencia significativa entre los grupos es en el estado civil $(F=32,402 ; p<0,001)$, con una mayor incidencia de casados en el grupo de control sano y de divorciados en el grupo de pacientes dependientes del alcohol. Los dos grupos de pacientes tienen una edad media de en torno a 40 años, cuentan con un nivel cultural y social medio y hay un predominio de hombres sobre mujeres, pero no hay diferencias entre uno y otro grupo.

\section{Características psicopatológicas}

Como puede apreciarse en la tabla 2, existen diferencias estadísticamente significativas en cuanto a las características psicopatológicas. Por lo que se refiere a la psicopatología global, aparecen diferencias estadísticamente significativas entre los dos grupos de pacientes y el grupo de control sano en el SCL-90-R (reflejadas en el Índice Global de Severidad). Es decir, la sintomatología psicopatológica general es mayor en ambos grupos de pacientes que en el grupo de control sano, pero sin diferencias entre uno y otro grupo.

Respecto a las características psicopatológicas más específicas, la sintomatología ansioso-depresiva es mucho más alta en los grupos de pacientes que en el grupo de control sano. Tampoco en este caso hay diferencias entre los dos grupos de pacientes.

En cuanto a la adaptación a la vida cotidiana, los pacientes de ambos grupos de pacientes están peor adaptados que el grupo de control sano. Una vez más, no hay diferencias entre uno y otro grupo de pacientes. 
Tabla 1. Variables sociodemográficas de la muestra

\begin{tabular}{|c|c|c|c|c|}
\hline Variables & $\begin{array}{c}\text { Grupo } \\
\text { experimental de alcohólicos } \\
\mathrm{N}=158 \\
\text { Media (DT) }\end{array}$ & $\begin{array}{l}\text { Grupo de control clínico } \\
\qquad \begin{array}{c}\mathrm{N}=120 \\
\text { Media (DT) }\end{array}\end{array}$ & $\begin{array}{l}\text { Grupo de control sano } \\
\qquad \begin{array}{c}\mathrm{N}=103 \\
\text { Media (DT) }\end{array}\end{array}$ & \\
\hline Edad Media & $43,42(12,011)$ & $40,58(13,350)$ & $40,73(11,343)$ & \\
\hline Variables & $\begin{array}{c}\text { Grupo } \\
\text { experimental de alcohólicos } \\
N=158 \mathrm{~N}(\%)\end{array}$ & $\begin{array}{l}\text { Grupo de control clínico } \\
\qquad N=120 N(\%)\end{array}$ & $\begin{array}{l}\text { Grupo de control sano } \\
\qquad N=103 N(\%)\end{array}$ & $\chi^{2}$ \\
\hline $\begin{array}{l}\text { Sexo } \\
\text { Hombre } \\
\text { Mujer }\end{array}$ & $\begin{array}{l}103(65,2 \%) \\
55(34,8 \%)\end{array}$ & $\begin{array}{l}64(53,3 \%) \\
56(46,7 \%)\end{array}$ & $\begin{array}{l}55(53,4 \%) \\
48(46,6 \%)\end{array}$ & 5,319 \\
\hline $\begin{array}{l}\text { Estado Civil } \\
\text { Soltero } \\
\text { Casado/conviviendo } \\
\text { Separado/divorciado } \\
\text { Viudo }\end{array}$ & $\begin{array}{c}57(36,1 \%) \\
61(38,6 \%) \\
32(20,3 \%) \\
8(5,1 \%) \\
\end{array}$ & $\begin{array}{c}55(45,8 \%) \\
47(39,2 \%) \\
12(10 \%) \\
6(5 \%) \\
\end{array}$ & $\begin{array}{c}31(30 \%) \\
67(65 \%) \\
4(3,9 \%) \\
1(1 \%) \\
\end{array}$ & $32,402 * * *$ \\
\hline $\begin{array}{l}\text { Nivel Cultural } \\
\text { Sin estudios } \\
\text { Estudios primarios } \\
\text { Estudios secundarios } \\
\text { Universidad }\end{array}$ & $\begin{array}{c}7(4,4 \%) \\
41(25,9 \%) \\
63(39,9 \%) \\
47(29,7 \%)\end{array}$ & $\begin{array}{c}4(3,3 \%) \\
33(27,5 \%) \\
49(40,8 \%) \\
34(28,3 \%)\end{array}$ & $\begin{array}{c}10(9,7 \%) \\
22(21,4 \%) \\
32(31,1 \%) \\
39(37,9 \%)\end{array}$ & 9,118 \\
\hline $\begin{array}{l}\text { Nivel Socioeconómico } \\
\text { Medio bajo } \\
\text { Medio } \\
\text { Medio alto }\end{array}$ & $\begin{array}{l}18(11,4 \%) \\
111(70,3 \%) \\
29(18,4 \%)\end{array}$ & $\begin{array}{l}19(15,8 \%) \\
77(64,2 \%) \\
24(20 \%)\end{array}$ & $\begin{array}{l}12(11,7 \%) \\
76(73,8 \%) \\
15(14,6 \%)\end{array}$ & 2,940 \\
\hline
\end{tabular}

*** $p<0,001$. DT=desviación típica

Tabla 2. Características psicopatológicas de los grupos estudiados

\begin{tabular}{|c|c|c|c|c|}
\hline CARACTERÍSTICAS PSICOPATOLÓGICAS & $\begin{array}{c}\text { Grupo experimental } \\
\text { de alcohólicos } \\
\text { X (DT) }\end{array}$ & $\begin{array}{l}\text { Grupo de control } \\
\text { clínico } \\
\text { X (DT) }\end{array}$ & $\begin{array}{c}\text { Grupo de control sano } \\
\text { X (DT) }\end{array}$ & $\mathbf{F}$ \\
\hline $\begin{array}{l}\text { SCL-90-R } \\
\text { (Rango: 0-100) } \\
\text { Somatización } \\
\text { Obsesión-compulsión } \\
\text { Sens. Interpersonal } \\
\text { Depresión } \\
\text { Ansiedad } \\
\text { Hostilidad } \\
\text { Ansiedad fóbica } \\
\text { Ideación paranoide } \\
\text { Psicoticismo } \\
\text { GSI } \\
\text { PST } \\
\text { PSDI }\end{array}$ & $\begin{array}{l}42,8(23,3) \\
55,8(24,9) \\
48,4(24,2) \\
57,2(21) \\
52,1(23,3) \\
49,1(30) \\
48,4(27,8) \\
54,2(25,8) \\
64,2(20,2) \\
55,3(20,6) \\
57,9(22) \\
48,3(21,5)\end{array}$ & $\begin{array}{l}45,3(27,4) \\
59,9(22) \\
55,4(25,8) \\
63,9(23,7) \\
56,4(25,1) \\
48,3(30,4) \\
52,8(29,2) \\
51,4(26,2) \\
62,4(20,5) \\
60,1(21,4) \\
60(22,7) \\
54,6(22,7)\end{array}$ & $\begin{array}{l}39,5(20,6) \\
51,5(20,8) \\
49,4(19,5) \\
38,6(20) \\
40,1(21,3) \\
46,4(22,5) \\
42,6(20,2) \\
51,6(20,5) \\
51,3(24,4) \\
45,3(16,8) \\
60,9(20,7) \\
23,2(16,1)\end{array}$ & $\begin{array}{c}1,09 \\
2,51 \\
2,08 \\
27,91 * * * \\
9,92 * * * \\
0,19 \\
2,83 \\
0,35 \\
8,28 * * * \\
11,13 * * * \\
0,44 \\
50,62 * * *\end{array}$ \\
\hline Ansiedad ${ }^{1}$ (STAI-E) (Rango: 0-100) & $54,4(28,8)$ & $63,9(29,2)$ & $39,1(22,4)$ & $16,72^{* * *}$ \\
\hline Depresión (BDI) (Rango: 0-63) & $19,7(8)$ & $22,4(10)$ & $6,9(5,9)$ & $80,57^{* * *}$ \\
\hline Inadaptación (EI) (Rango: 0-30) & $19,1(6,4)$ & $18,7(6,8)$ & $6,9(5,6)$ & $90,73^{* * *}$ \\
\hline
\end{tabular}

${ }^{* * *} p<0,001 .{ }^{1}$ En este caso las puntuaciones que figuran corresponden a los centiles

$X=$ media

DT=desviación típica

SCL-90-R: Listado de síntomas

STAI-E: Inventario de Ansiedad-Estado

BDI: Inventario de Depresión de Beck

El: Escala de Inadaptación 
Tabla 3. Dimensiones de personalidad de los grupos estudiados

\begin{tabular}{|c|c|c|c|c|}
\hline DIMENSIONES DE PERSONALIDAD & $\begin{array}{c}\text { Grupo experimental } \\
\text { de alcohólicos } \\
\text { X (DT) }\end{array}$ & $\begin{array}{l}\text { Grupo de control } \\
\text { clínico } \\
\text { X (DT) }\end{array}$ & $\begin{array}{l}\text { Grupo de control } \\
\text { sano } \\
\text { X (DT) }\end{array}$ & $\mathbf{F}$ \\
\hline $\begin{array}{l}\text { Ansiedad }{ }^{1} \text { (STAI-R) } \\
\text { (Rango: 0-100) }\end{array}$ & $75,6(27,2)$ & $76,7(28,8)$ & $34,5(20)$ & $74,88 * * *$ \\
\hline $\begin{array}{l}\text { Impulsividad (BIS-10) } \\
\text { (Rango: 0-132) } \\
\text { Impulsividad motora } \\
\text { Impulsividad cognitiva } \\
\text { Improvisación y ausencia de planificación }\end{array}$ & $\begin{array}{l}61(18,5) \\
20,1(7,3) \\
21,9(6,2) \\
19,4(8,2)\end{array}$ & $\begin{array}{l}55(18) \\
17,1(8,4) \\
21,3(5,3) \\
16,7(7,8)\end{array}$ & $\begin{array}{l}45(12,6) \\
13,2(5,9) \\
17,2(4,6) \\
15,4(4,3)\end{array}$ & $\begin{array}{c}18,81^{* * *} \\
18,14^{* * *} \\
16,96^{* * *} \\
7,03^{* *}\end{array}$ \\
\hline $\begin{array}{l}\text { Búsqueda de sensaciones (SSS) } \\
\text { (Rango: 0-40) }\end{array}$ & $20,2(6,7)$ & $16,5(7,3)$ & $16,6(4,8)$ & $8,67^{* * *}$ \\
\hline Búsqueda de emociones & $5,3(3,1)$ & $4,3(3,7)$ & $4,3(2)$ & 3,13 \\
\hline Búsqueda de excitación & $5,7(2,1)$ & $5,3(1,9)$ & $5,2(1,7)$ & 1,67 \\
\hline Desinhibición & $4,7(2,4)$ & $2,9(2,3)$ & $4,1(1,5)$ & $13,39 * * *$ \\
\hline Susceptibilidad al aburrimiento & $4,5(2)$ & $4,3(2)$ & $3,1(1,7)$ & $11,83^{* * *}$ \\
\hline
\end{tabular}

${ }^{*} p<0,05 ;{ }^{* *} p<0,01 ;{ }^{* * *} p<0,001$

1 En este caso las puntuaciones que figuran corresponden a los centiles

$X=$ media

DT=desviación típica

STAI: Inventario de Ansiedad

BIS-10: Escala de Impulsividad

SSS: Escala de Búsqueda de Sensaciones

\section{Dimensiones de personalidad}

En cuanto a las dimensiones de personalidad, los resultados aparecen descritos en la tabla 3. Los dos grupos de pacientes son más ansiosos que el grupo de control sano, pero no hay diferencias entre ellos.

Respecto a la impulsividad, los pacientes dependientes del alcohol muestran una mayor impulsividad motora y son más improvisadores y carentes de planificación que el otro grupo de pacientes (Bonferro$\mathrm{ni}=3,04$ y 2,74 , respectivamente) y que el grupo de control sano (Bonferroni=6,89 y 4,05, respectivamente). Asimismo el grupo de pacientes dependientes del alcohol presenta una mayor búsqueda de sensaciones y una mayor desinhibición que el otro grupo de pacientes (Bonferroni=3,61 y 1,71, respectivamente).

\section{Trastornos de personalidad}

Por lo que se refiere a los trastornos de personalidad reflejados en el MCMI-II, en líneas generales los dos grupos de pacientes presentan más trastornos que el grupo de control sano, pero sin diferencias entre ellos. Una excepción la constituye el trastorno compulsivo, que aparece presente de forma similar en los tres grupos (tabla 4 y figura 1).

Respecto a los dos grupos de pacientes, el grupo de pacientes dependientes del alcohol presenta una mayor incidencia de rasgos histriónicos (Bonferro$\mathrm{ni}=12,94$ ), narcisistas (Bonferroni $=10,62$ ) y antisociales (Bonferroni=17,78), que son los más específicos de este grupo. 
Tabla 4. Trastornos de personalidad según el MCMI-II en los grupos estudiados

\begin{tabular}{|c|c|c|c|c|c|c|c|}
\hline \multirow[t]{2}{*}{$\begin{array}{l}\text { TRASTORNOS DE } \\
\text { PERSONALIDAD } \\
\text { MCMI-II }\end{array}$} & \multicolumn{2}{|c|}{$\begin{array}{c}\text { Grupo experimental } \\
\text { de alcohólicos } \\
\text { N }=158\end{array}$} & \multicolumn{2}{|c|}{$\begin{array}{l}\text { Grupo de control clínico } \\
\qquad N=120\end{array}$} & \multicolumn{2}{|c|}{$\begin{array}{l}\text { Grupo de control sano } \\
\qquad N=103\end{array}$} & \multirow[t]{2}{*}{$F$} \\
\hline & $x$ & DT & $x$ & DT & $x$ & DT & \\
\hline Esquizoide & 62,8 & 27,6 & 70,3 & 27,6 & 38,4 & 27,6 & $40,29 * * *$ \\
\hline Fóbico & 54,2 & 25,0 & 54,2 & 29,8 & 21 & 17,2 & $66,50 * * *$ \\
\hline Dependiente & 68,5 & 30,1 & 66,7 & 32,2 & 48,9 & 26,7 & $14,91 * * *$ \\
\hline Histriónico & 63,6 & 27,7 & 50,6 & 31,2 & 50,5 & 25,8 & $9,68 * * *$ \\
\hline Narcisista & 67,4 & 29,2 & 56,8 & 31,1 & 51,8 & 26,0 & $9,95 * * *$ \\
\hline Antisocial & 67,5 & 28,3 & 49,7 & 29,9 & 40 & 23,2 & $33,62 * * *$ \\
\hline Agresivo-sádico & 63 & 29,7 & 56,4 & 29,5 & 39,1 & 22,7 & $23,11 * * *$ \\
\hline Compulsivo & 73,9 & 31,7 & 80,8 & 32,1 & 76,8 & 29,3 & 1,67 \\
\hline Pasivo-agresivo & 57,4 & 26,9 & 51,1 & 31,4 & 27,3 & 17,3 & $42,44^{* * *}$ \\
\hline Autodestructivo & 59,8 & 25,3 & 54,7 & 26,9 & 21,4 & 19,3 & $84,09 * * *$ \\
\hline Esquizotípico & 59,3 & 24,6 & 64,5 & 28,4 & 31,5 & 17,3 & $59,20 * * *$ \\
\hline Límite & 63,6 & 25,8 & 59,8 & 28,5 & 29 & 17,1 & $67,41 * * *$ \\
\hline Paranoide & 70,6 & 27,5 & 66,2 & 32,4 & 43,3 & 27,1 & $29,50 * * *$ \\
\hline
\end{tabular}

*** $p<0,001$

$X=$ media

DT=desviación típica

MCMI: Inventario Clínico Multiaxial de Millon

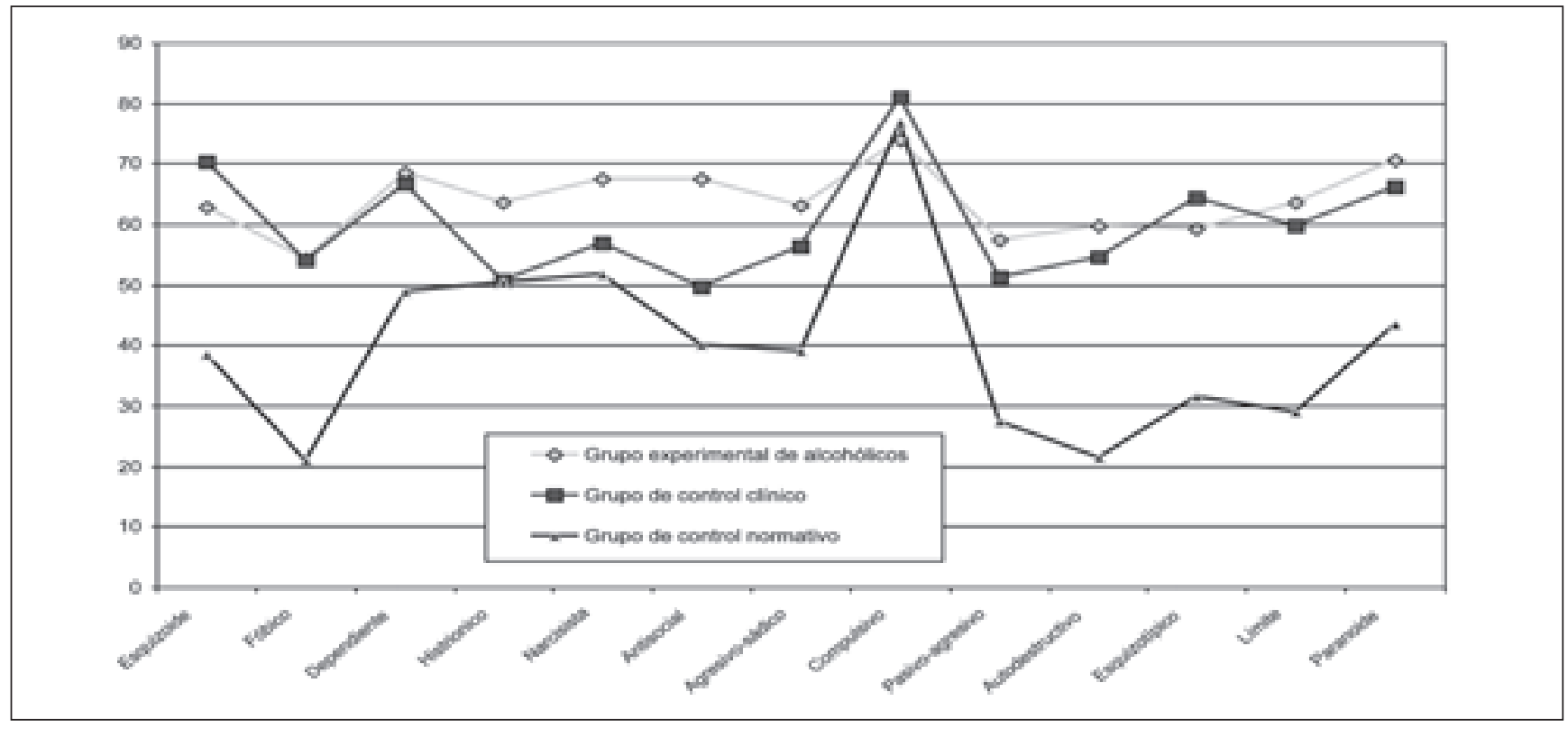

Figura 1. Rasgos diferenciales de personalidad según el MCMI-II en los grupos estudiados

\section{DISCUSIÓN}

Aun siendo abundante la bibliografía sobre la dependencia del alcohol, es relativamente escasa la que enfoca este problema en función de las dimensiones de personalidad de los pacientes y de la comorbilidad psicopatológica planteada. Este estudio supone una clarificación de la comorbilidad psicopa- tológica de la dependencia del alcohol, así como un mayor conocimiento de los rasgos y de los trastornos de personalidad implicados, y da pie para futuros estudios relacionados con programas de intervención diferenciados para este tipo de pacientes.

Respecto a las características demográficas, la inestabilidad familiar (la tasa de divorcios) es mayor 
en el grupo de pacientes dependientes del alcohol que en el resto de los pacientes y que en el grupo de control sano. En ambos grupos de pacientes (dependientes del alcohol y no-adictos) la sintomatología psicopatológica, especialmente la de tipo ansioso-depresiva, es abundante, lo que genera una mala adaptación a la vida cotidiana. Todo ello es coincidente con estudios previos (Landa et al., 2006; Sanchéz Hervás, Tomás Gradoli y Morales Gallús, 2001; Ross, 1995). Como era previsible, el grupo de control sano presenta menor sintomatología psicopatológica y está mejor adaptado a la vida cotidiana.

En cuanto a las dimensiones de personalidad, uno y otro tipo de pacientes son más ansiosos que el grupo de control sano. Por lo que se refiere al perfil de los pacientes dependientes del alcohol, éstos tienden a ser más impulsivos y buscadores de sensaciones que el resto de los pacientes y de los sujetos sanos, como ocurre también en otro tipo de adictos (Fernández-Montalvo, Echeburúa y Báez, 1999).

Como era de esperar, los trastornos de personalidad son más frecuentes en los dos grupos de pacientes que en el grupo de control sano, excepto en lo referido al rasgo obsesivo-compulsivo de la personalidad, que aparece de forma similar en los tres grupos. Se trata, por tanto, de un rasgo de personalidad muy presente en la población general. En el grupo de pacientes dependientes del alcohol hay una mayor incidencia de rasgos histriónicos, narcisistas y antisociales, lo que coincide sólo parcialmente con otros estudios (Echeburúa et al., 2005, 2007; FernándezMontalvo y Landa, 2003; Regier, Farmer, Rae, Locke, Keith, Judd y Goodwin, 1990).

En síntesis, a partir de los resultados expuestos, se puede concluir que los pacientes dependientes del alcohol tienen una peor adaptación familiar, presentan muchos síntomas ansioso-depresivos y tienden a ser impulsivos y buscadores de sensaciones, con rasgos de personalidad histriónicos, narcisistas y antisociales. Convendría, por ello, desarrollar programas de tratamiento específicos y diseños de intervención que cuenten con estos aspectos para lograr una mayor eficacia terapéutica.

Este estudio tiene un carácter exploratorio. Por ello, algunas limitaciones de este trabajo son el recurso a una muestra de pacientes dependientes del alcohol que están en tratamiento, lo que limita las conclusiones epidemiológicas obtenidas, y el enfoque categorial dado a los trastornos de personalidad en función del instrumento utilizado. Otros estudios futuros deberían abordar estas limitaciones.

En conclusión, y a pesar de las limitaciones señaladas, este estudio aporta un enfoque novedoso que requiere continuidad en investigaciones futuras, especialmente por lo que se refiere al diseño de programas de intervención de pacientes dependientes del alcohol más específicos en función del perfil psicopatológico analizado.

\section{REFERENCIAS}

American Psychiatric Association (2000). Diagnostic and statistical manual of mental disorders (4th ed. revised). Washington, DC: APA.

Barratt, E. S. (1985) Impulsiveness defined within a systems model of personality. En E. P. Spielberger y J. N. Butcher (Eds.), Advances in Personality Assessment (pp. 113-132). Hillsdale: Lawrence Erlbaum Associates.

Beck, A. T., Steer, R. A. y Garbin, M. G. (1988). Psychometrics properties of the Beck's depression inventory. Twentyfive years of evaluation. Clinical Psychology Review, 8, 77-100.

Beck, A. T., Rush, A. J., Shaw, B. F. y Emery, G. (1979). Cognitive therapy of depression. New York: Guilford Press.

Bravo de Medina, R., Echeburúa, E. y Aizpiri, J. (en prensa). Diferencias de sexo en el alcoholismo: dimensiones de personalidad, características psicopatológicas y trastornos de personalidad. Psicothema.

Casas, M. (1994). Psicopatología y alcoholismo. Barcelona: Citrán.

Casas, M. y Guardia, J. (2002). Patología psiquiátrica asociada al alcoholismo. Adicciones, 14, 195-219.

Derogatis, L. R. (1975). The SCL-90-R. Baltimore: Clinical Psychometric Research.

Echeburúa, E., Bravo de Medina, R. y Aizpiri, J. (2005). Alcoholism and personality disorders: An exploratory study. Alcohol \& Alcoholism, 40, 323-326.

Echeburúa, E., Bravo de Medina, R. y Aizpiri, J. (2007). Comorbidity of alcohol dependence and personality disorders: A comparative study. Alcohol \& Alcoholism, 42.

Echeburúa, E., Corral, P. y Fernández-Montalvo, J. (2000). Escala de inadaptación (El): Propiedades psicométricas en contextos clínicos. Análisis y Modificación de Conducta, 26, 325-340.

Fernández-Montalvo, J. y Landa, N. (2003). Comorbilidad del alcoholismo con los trastornos de personalidad. Clínica y Salud, 14, 27-41.

Fernández-Montalvo, J., Echeburúa, E. y Báez, C. (1999). Variables de inteligencia y personalidad en los jugadores patológicos de máquinas tragaperras: un estudio descriptivo. Psicología Conductual, 7, 349-360.

Fernández-Montalvo, J., Landa, N. y López-Goñi, J. J. (2005). Prevalencia del juego patológico en el alcoholismo: un estudio exploratorio. Revista de Psicopatología y de Psicología Clínica, 10, 125-134.

González de Rivera, J. L. (2002). Versión española del SCL90-R. Madrid: TEA. 
Haver, B. (2003). Comorbid psychiatric disorders predict and influence treatment outcome in female alcoholics. European Addictions Research, 9, 39-44.

Holdcraft, L. C., Jacono, W. G. y McGue, M. K. (1998). Antisocial personality disorder and depression in relation to alcoholism: A community-based sample. Journal of Studies of Alcohol, 59, 222-226.

Kranzler, H. R., Mason, B. y Modesto-Lowe, V. (1998). Prevalence, diagnosis and treatment of comorbid mood disorders and alcoholism. En H. R. Kranzler y B. Rousanville (Eds.), Dual Diagnosis and Treatment (pp. 107-136). New York: Marcel Dekker, Inc.

Landa, N., Fernández.Montalvo, J., López-Goñi, J. J. y Lorea, I. (2006). Comorbilidad psicopatológica en el alcoholismo: un estudio descriptivo. International Journal of Clinical and Health Psychology, 6, 253-269.

Luengo, M. A., Carrillo de la Peña, M. T. y Otero, J. M. (1991). The components of impulsiveness: a comparison of the 1.7 Impulsiveness Questionnaire and the Barratt Impulsiveness Scale. Personality and Individual Differences, 12, 657-667.

Millon, T. (1997). Millon Clinical Multiaxial Inventory-II (MCMIII). Minneapolis. National Computer Systems.

Pérez, J. y Torrubia, R. (1986). Fiabilidad y validez de la versión española de la escala de búsqueda de sensaciones (forma V). Revista Latinoamericana de Psicología, 18, 7-22.

Regier, D. A., Farmer, M. E., Rae, D. S., Locke, B. Z., Keith, S. J., Judd, L. L. y Goodwin, F. K. (1990). Comorbidity of mental disorders with alcohol and other drug abuse. Results from the Epidemiological Cathment Area (ECA) Study. Journal of the American Medical Association, 21, 2511-2518.

Richter, P., Werner, J., Heerlein, A., Jraus, A. y Sauer, $H$. (1989). On the validity of the Beck Depression Inventory. A review. Psichopathology, 3, 160-168.

Ross, H. E. (1995). DSM-III-R alcohol abuse and dependence and psychiatric comorbidity in Ontario: Results From the Mental Health Supplement to the Ontario Health Survey. Drug and Alcohol Dependence, 39, 111-128.

Rousanville, M. D., Dolinsky, Z. S., Babor, T. F. y Meyer, R. (1987). Psychopathology as a predictor of treatment outcome in alcoholics. Archives of General Psychiatry, 44, 505-513.

Sánchez Hervás, E., Tomás Gradoli, V. y Morales Gallús, E. (2001). Evaluación psicopatológica en dependientes a alcohol, heroína y cocaína mediante el Brief Symptom Inventory. Adicciones, 13, 61-66.

Sanz, J. y Vázquez, C. (1998). Fiabilidad, validez y datos normativos del inventario para la depresión de Beck. Psicothema, 2, 303-318.

Sanz, J. y Vázquez, C. (1998). Fiabilidad, validez y datos normativos del inventario para la depresión de Beck. Psicothema, 2, 303-318.

Spielberger, C. D., Gorsuch, R. L. y Lushene, R. (1982). Manual del Cuestionario de Ansiedad Estado/Rasgo (STAl) (3 ${ }^{a}$ edic.). Madrid: TEA.

Spielberger, C. D., Gorsuch, R. L. y Lushene, R. E. (1970). STAl Manual for the State-Trait Anxiety Inventory (Self-Evaluation Questionnaire). Palo Alto California: Consulting Psychologists Press.

Vázquez, C. y Sanz, J. (1999). Fiabilidad y validez de la versión española del Inventario para la Depresión de Beck de 1978 en pacientes con trastornos psicológicos. Clínica y Salud, 10, 59-81.

Weis, R. D., Griffin, M. L. y Mirin, S. M. (1989). Diagnosing major depression in cocaine abusers: The use of depression rating scales. Psychiatry Research, 3, 335-343.

Weltzer, S. (1990). The Millon Clinical Multiaxial Inventory (MCMI): A review. Journal of Personality Assesment, 55, 445-464.

Zuckerman, M., Eysenck, S. B. y Eysenck, A. J. (1978). Sensation Seeking in England and American: crosscultural age and sex comparisons. Journal of Consulting and Clinical Psychology, 46, 139-149. 
